\title{
Acute and Chronic Periocular Massage for Ocular Blood Flow and Vision: a Randomized Controlled Trial
}

Naoyuki Hayashi, PhD,, $2^{*}$ and Lanfei Du, $\mathrm{MSc}^{2}$

IInstitute for Liberal Arts, Tokyo Institute of Technology, Meguro, Tokyo; ${ }^{2}$ Department of Social and Human Sciences, Tokyo Institute of Technology, Meguro, Tokyo, Japan

Introduction: The ocular blood flow (OBF) is responsible for supplying nutrition to the retina, which plays a fundamental role in visual function. Massage is expected to improve the blood flow and, consequently, vascular function. The aim of this study was to determine the shortterm and long-term effects of periocular massage on OBF and visual acuity.

Methods: The OBF and visual acuity were measured in 40 healthy adults aged 20-30 years before and after massage, and also in control subjects. Three massage methods were used: applying periocular acupressure ("Chinese eye exercise": CE), using a facial massage roller (MR), and using an automated eye massager (AM). The OBF and visual acuity were first measured before and after applying each type of massage for $5 \mathrm{~min}$. Eye massage was then applied for $5 \mathrm{~min}$ once daily over a 60-day period, while the control group received no massage. The same measurements were then performed again.

Results: Performing short-term periocular massage showed significant interactions in time and massage effects on visual acuity in CE and AM groups, and on OBF in AM group, while 60-day massage period exerted no significant effects. No significant relationship was found between OBF and visual acuity changes.

Conclusions: These results suggest that short-term periocular massage with Chinese eye exercise and automated eye massager can improve OBF and visual acuity, although no causal relationship was supported.

KEYWORDS: periocular massage; ocular blood flow; visual acuity, contrast sensitivity, adult subject

\section{INTRODUCTION}

Myopia is a worldwide public health issue, particularly in Asia. Children in Asian populations are indicated to be more susceptible to myopia, compared with Western populations. $(1,2,3)$ The prevalence rates of myopia in adults in Japan and China were reported to be $50 \%$ and $29 \%$, respectively. The prevalence of low vision was reported to be as high as $1 \%$ in more than 6,000 urban Chinese, and this was related to ageing. (4) Myopic retinopathy occurs in roughly $40 \%$ of highly myopic eyes ${ }^{(5)}$ and is a common cause of visual impairment. ${ }^{(5)}$ Several treatment options such as contact lenses and pharmaceutical agents have been devised, ${ }^{(6,7)}$ but methods for effectively and easily preventing myopia need to be developed.

Periocular massage has been used for maintaining vision. Since 1963, Chinese children in primary and junior middle schools have been required to perform 5-minute eye exercises every day for protecting vision and preventing myopia, but the effects of this massage are still unclear. (8) Previous studies of periocular massage and acupressure (i.e., Chinese eye exercise) found solely subjective changes or only small or modest objective changes in vision. $(8,9,10,11)$ However, those studies recruited children and, therefore, it is likely that the effects of growth masked the effects of massage. (9)

The short-term effects of massage have not been investigated, despite temporary effects also being useful for improving the quality of life. The spread of SARS-CoV-2 infections in 2020 has greatly restricted people movements worldwide, unavoidably resulting in longer times being spent watching television and participating in online gaming and social networking.(12) The known effects associated with this 
behaviour include eye strain. It is therefore prudent to consider the effects of periocular massage on visual function, and particularly short-term massage in adults.

Structural and functional impairments in the ocular vasculature increase the risk of vision impairment. $13,14,15)$ Eye diseases such as diabetic retinopathy, glaucoma, and age-related macular degeneration are relatively common in older populations. Preventing ocular vascular dysfunction can improve the quality of life. The ocular blood flow (OBF) supplies nutrients to the retina, which plays a fundamental role in vision. ${ }^{(16)}$ Intervening in the arterial $\mathrm{CO}_{2}$ partial pressure empirically revealed that changes in OBF are associated with changes in visual acuity. ${ }^{(17)}$ Also, many causes of blindness are due to abnormalities of the fundus circulation. Improving OBF could, therefore, help to prevent eye diseases and myopia.

Massage has been suggested to improve peripheral vessel function and blood flow, but such an effect has not been reported previously in the ocular region. Massage restores peripheral vascular function after exercise-induced muscle injury in sedentary young adults. (18) Acute massage applied using a roller increased the skin blood flow, and chronic massage improved the blood flow and its response to heat stimulation, probably resulting from improvements in vessel function. ${ }^{19)}$ Physical stimuli, such as pressure and abrasion, also increase the blood flow. (20) These findings suggest that massage applied to periocular regions can improve OBF.

Any increase in OBF induced by massage may improve vision. It is therefore worth examining whether periocular massage increases OBF and, consequently, improves vision. In this study we examined the shortterm and long-term effects of periocular massage on OBF and visual function using three massage methods that are known to have few side effects: Chinese eye exercise, manual massage using a facial massage roller, and an automated eye massager. The effects of these types of massage were examined by comparing with a control group or a control eye.

\section{METHODS}

\section{Ethics Approval and Trial Registration}

The experiments were approved by the ethics committee of the Tokyo Institute of Technology, Japan (approval number: 2017107), and registered by UMIN Clinical Trials Registry, UMINOOOO34654. Registered 26 October 2018, https://upload.umin.ac.jp/cgi-open-bin/ctr_e/ctr_view. cgi?recptno=R000039509

\section{Subjects}

Twenty males and twenty females aged $23.6 \pm 2.3$ years (mean \pm SD) volunteered in both the acute and chronic trials. Each subject was a graduate or undergraduate student. Twenty-one of them were from China, 17 were from Japan, and two were from other Asian countries. All of the subjects were free of any known ophthalmic diseases, autonomic dysfunction, and cardiovascular diseases, and were not taking any medications. Each subject received verbal and written explanations of the objectives, measurement techniques, and risks and benefits associated with the study, and they then provided written informed consent. Subjects were requested to abstain from consuming caffeinated beverages and eating for at least 2 hrs prior to the measurements in both experiments. All of the applied protocols conformed with the standards set by the Declaration of Helsinki.

All subjects were included in both the acute and chronic experiments, and were allocated to the four trial groups in a randomized manner: Chinese eye exercise (CE), facial massage roller (MR), automated eye massager (AM), and no-massage (control; CT) groups. Ten subjects were allocated to each group (Figure 1).

\section{Experimental Protocol and Measurements}

\section{Periocular massage}

The duration of each session of periocular massage was $5 \mathrm{~min}$ in both the acute and chronic experiments. In the CE group, the exercise was performed following instructions from a Chinese researcher who was experienced in "Chinese eye exercise". Locations of acupoints of standard Chinese eye exercises and information on how to massage were provided as in previous studies. ${ }^{(8,10)}$ In a preliminary trial, those who had never done this exercise tried it and they felt unnatural to do it with one eye only. Then we used both eyes for Chinese eye exercise and recruited other subjects as a control group. In the AM group, massage was applied using a battery-powered massager (Dreamin EYE 


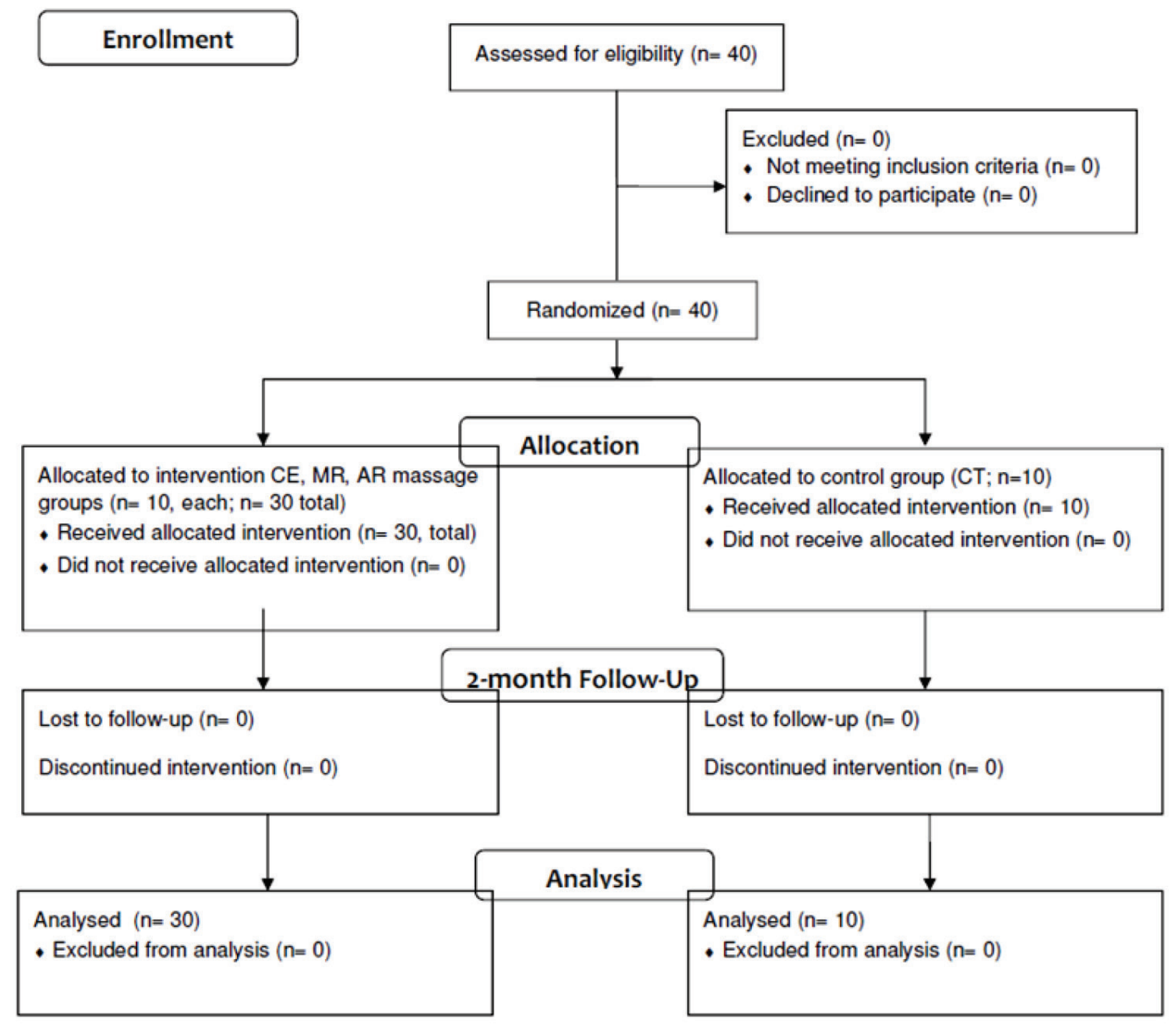

FIGURE 1. CONSORT flow diagram.

DR-ES2015B, MTG Co. Ltd., Nagoya, Japan). Airbags placed around both eyes and temples areas apply massage around both eyes. In the control (CT) group, no massage was applied around the eyes. In the MR group, massage was manually performed by applying a facial massage roller (ReFa CARAT Face, MTG) around the subject's right eye at a comfortable intensity, speed and frequency, while massage was not applied to the left eye. Data obtained from the left eye was used as the control solely in MR group.

\section{Acute experiment}

Before starting the measurements, each subject rested in a seated position for
20 min in an air-conditioned room maintained at $22^{\circ} \mathrm{C}$ to ensure temperature acclimation. After the resting period, the OBF, static visual acuity, and contrast sensitivity were recorded. The allocated massage was then applied to the subject for $5 \mathrm{~min}$, and the recordings were repeated immediately after the massage.

\section{Chronic experiment}

After the acute experiment was completed, the subjects received 5 min of massage using a given method every day for 60 days. One day after finishing the last massage, the baseline measurements performed before the massage in the acute experiment were repeated. 
The participants, other than those in the CT group, were told that if they could not receive massage for a total of at least 10 days, their participation was terminated. Subjects recorded their massage time every day, and an experimenter checked these records when performing the measurements after the intervention. All of the participants kept appropriate records.

\section{Ocular blood flow recording}

Subjects were asked to keep their face motionless in front of a laser-speckle flowgraphy (LSFG) apparatus (LSFG-NAVI, SoftCare, Fukuoka, Japan) while LSFG images were obtained. Subjects were asked to open their right eye without blinking for $4 \mathrm{sec}$ while the images were recorded for measuring the OBF, with three images being obtained. Subjects who normally wore glasses or contact lenses removed them before the experiment. No drugs, such as those for mydriasis, were administered during the experiments.

LSFG assesses the mean blur rate (MBR), which reflects the velocity of the OBF and is known to be correlated with the actual blood flow volume as measured using hydrogen gas clearance and microsphere methods. ${ }^{(21,22)}$ We assessed the blood flow profile using LSFG Analyser software (version 3.0.47, SoftCare). The analysis method was similar to those reported previously. ${ }^{(23,24)}$ The software program selected the optic nerve head using the automated definitive threshold (Figure 2). The following indices were calculated from the obtained data: MBR which reflects the velocity of the OBF, blow-out time (BOT), and blow-out score (BOS).

BOT and BOS have been proposed as indices of the OBF profile that can reflect

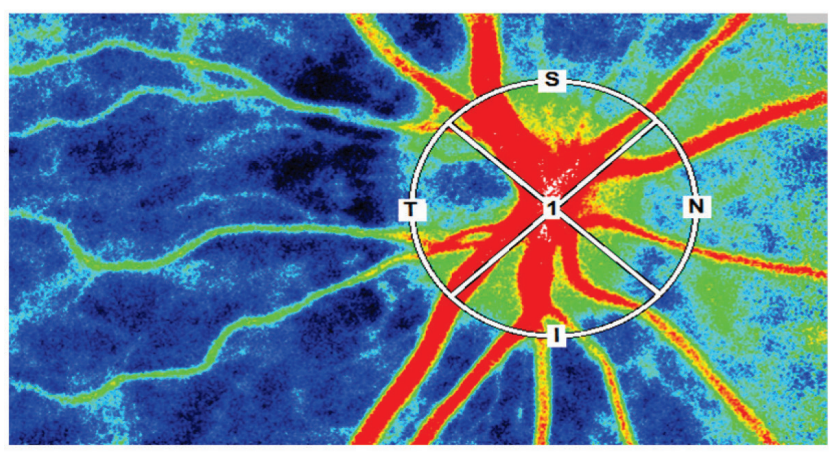

FIGURE 2. An example image of the inner ocular circulation obtained by laser-speckle flowgraphy; the white circle indicates the region of interest (the optic nerve head). vascular flexibility. (25) The BOT represents the proportion of time during a single heartbeat that the waveform is higher than half of the mean of the minimum and maximum signals. A high BOT indicates that a high level of blood flow is maintained for a relatively long time during each heartbeat, thus indicating that abundant nutrition is being supplied to the periphery. BOS is a value to evaluate the amount of blood flow during one heart cycle. The BOS is calculated using the difference between the maximum and minimum MBRs, as well as the average MBR. A high BOS indicates a high constancy of blood flow during each heartbeat.

\section{Visual acuity and contrast sensitivity recordings}

The contrast sensitivity was measured at $10 \%$ and $100 \%$ (visual acuity) using a contrast sensitivity and visual acuity tester (CAT-P, Neitz Instruments Co. Ltd., Tokyo, Japan). The decimal visual acuity value was measured using a Landolt ring for the target. Measurements were made twice for each eye and averaged. The decimal visual acuity value was converted to the logarithm of the minimum angle of resolution (log MAR) using the following formula: log MAR $=-\log \left(\right.$ decimal visual acuity). ${ }^{(26)}$

\section{Statistical Analysis}

All statistical analyses were performed with the R software (version 3.5.1). The probability criterion for statistical significance was set at $p<.05$. The results obtained in both experiments did not distinguish between males and females, since no sexrelated difference was found in the relative changes in the variables.

The effects of time (before vs. after massage) and intervention (massage vs. control) were tested using two-way repeated ANOVA to detect the effects of massage on $O B F$ and visual function. The effects of the different types of massage (i.e., in the CE, $M R$, and AM groups) were not examined since we were not attempting to identify the better type of massage. Therefore, the effects of each type of massage on OBF variables, visual acuity, and contrast sensitivity were independently tested against the CT group (CE and AM groups) or control eye (MR group). Pearson's correlation analysis was applied to pooled data of all massage groups $(n=30)$ to examine the relationship between the changes in OBF 
and visual function between before and after massage, and between the visual acuity before massage and the change in visual acuity induced by massage.

\section{RESULTS}

Demographic information of participants in each group is shown in Table 1.

\section{Acute Experiment}

The effects of $5 \mathrm{~min}$ of massage on OBF, visual acuity, and contrast sensitivity are summarized in Table 2. A significant main effect of time on visual acuity was observed in the CE, AM, and MR

TABLE 1. Demographic Information in Each Group

\begin{tabular}{lccc}
\hline & $M / F$ & Age $^{a}$ & Glasses/Contact \\
\hline CE & $5 / 5$ & $23 \pm 2.0$ & $6 / 3$ \\
AM & $5 / 5$ & $24 \pm 2.5$ & $6 / 1$ \\
MR & $5 / 5$ & $25 \pm 2.3$. & $5 / 1$ \\
CT & $5 / 5$ & $23 \pm 2.4$ & $5 / 2$ \\
\hline
\end{tabular}

aMean \pm SD of age

${ }^{b}$ Numbers of subjects wearing glasses or contact lenses.

$\mathrm{CE}=$ Chinese eye exercise; $\mathrm{AM}=$ automatic

massager; $\mathrm{MR}=$ facial massage roller; $\mathrm{CT}=$ control. groups, while a significant interaction of time and intervention on visual acuity was observed in the CE and AM groups (Figure 3). Supplemental paired tests of the pooled data obtained from the right eyes in the three massage groups $(n=30)$ showed significant differences between before and after massage (0.14 $\pm 0.20 \mathrm{vs}$. $0.056 \pm 0.190)$.

A significant interaction of group and intervention on the MBR of the OBF was observed in the AM and MR groups, while no main effect of time was observed. No significant correlation was observed in changes between the MBR and visual acuity either in the data for each group or in the pooled data of the three groups (Figure 4).

The relationship between the visual acuity before massage and the change in visual acuity induced by massage is shown in Figure 5. There was a tendency for a correlation $(p<.1)$. A significant main effect of time on the contrast sensitivity at 10\% was observed in the CE and AM groups, while no interaction effect was observed. No significant effect was observed for the other variables.

\section{Chronic Experiment}

None of the participants was lost to followup, and so all 40 participants were analyzed in the chronic experiment. According to the subjects' records, the number of days without massage was less than five.

TABle 2. Changes in Visual Functions and Ocular Circulation Before and After 5-Min Periocular Massage

\begin{tabular}{|c|c|c|c|c|c|c|}
\hline Group & & Visual Acuity & Cont. Sens.10\% & $M B R$ & BOS & BOT \\
\hline \multirow[t]{2}{*}{$C E^{a}$} & Before & $0.23 \pm 0.25$ & $0.24 \pm 0.18$ & $25.0 \pm 3.8$ & $79.5 \pm 4.1$ & $57.5 \pm 4.5$ \\
\hline & After & $0.16 \pm 0.24^{c d}$ & $0.27 \pm 0.19^{c}$ & $25.0 \pm 5.9$ & $80.1 \pm 4.5$ & $57.5 \pm 2.8$ \\
\hline \multirow[t]{2}{*}{$\mathrm{AM}^{\mathrm{a}}$} & Before & $0.06 \pm 0.10$ & $0.37 \pm 0.15$ & $27.0 \pm 5.5$ & $79.9 \pm 2.3$ & $58.1 \pm 4.3$ \\
\hline & After & $-0.04 \pm 0.05^{c d}$ & $0.49 \pm 0.21^{c}$ & $27.8 \pm 6.1^{d}$ & $79.6 \pm 3.5$ & $56.6 \pm 4.5$ \\
\hline \multirow[t]{2}{*}{$C T^{\mathrm{a}}$} & Before & $0.15 \pm 0.28$ & $0.29 \pm 0.21$ & $25.3 \pm 7.2$ & $81.1 \pm 2.8$ & $58.0 \pm 3.1$ \\
\hline & After & $0.20 \pm 0.32$ & $0.37 \pm 0.29$ & $24.7 \pm 6.9$ & $80.4 \pm 4.6$ & $57.0 \pm 2.1$ \\
\hline \multirow[t]{2}{*}{$M R^{b}$} & Before & $0.12 \pm 0.19$ & $0.35 \pm 0.21$ & $25.6 \pm 5.1$ & $80.9 \pm 2.8$ & $58.3 \pm 2.6$ \\
\hline & After & $0.05 \pm 0.18^{c}$ & $0.37 \pm 0.13$ & $26.3 \pm 5.0^{d}$ & $81.8 \pm 2.5$ & $58.1 \pm 3.5$ \\
\hline \multirow[t]{2}{*}{$\mathrm{MR}(\mathrm{Ctrl})^{\mathrm{b}}$} & Before & $0.18 \pm 0.30$ & $0.41 \pm 0.23$ & $27.6 \pm 5.3$ & $80.8 \pm 2.8$ & $58.0 \pm 2.5$ \\
\hline & After & $0.24 \pm 0.40$ & $0.43 \pm 0.28$ & $26.9 \pm 5.4$ & $81.1 \pm 2.3$ & $56.7 \pm 2.9$ \\
\hline
\end{tabular}

a In CE (Chinese eye exercise) and AM (automatic massager) groups, CT (control) was used as control intervention.

bIn MR (facial massage roller) group, control left eye (MR Ctrl) was used as control intervention.

cMain effect of time $(p<.05)$.

dinteraction of time and intervention $(p<.05)$.

Cont Sens = contrast sensitivity; $\mathrm{MBR}=$ mean blur rate (blood flow); BOS = blow-out score; BOT = blow-out time. 


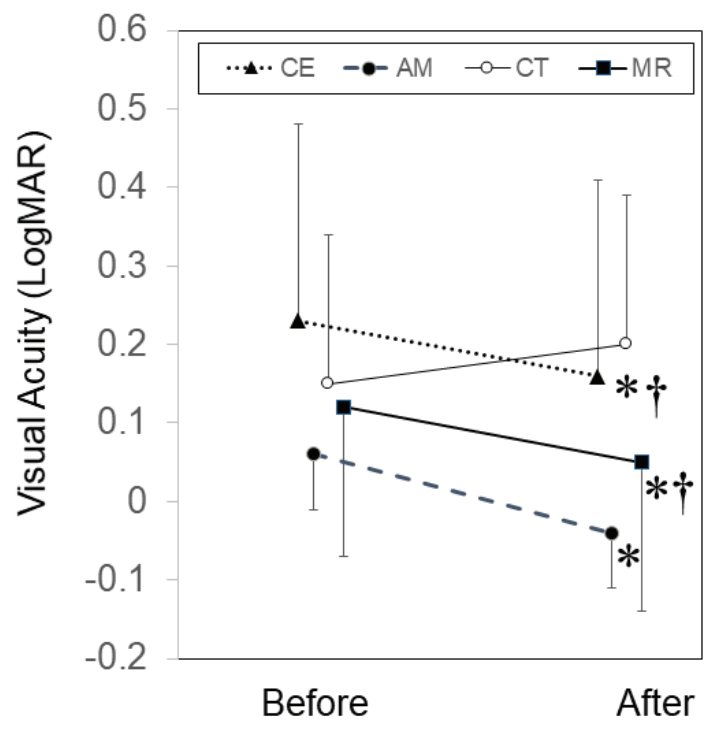

* = significant main effect of time; ${ }^{\dagger}=$ significant interaction of time and intervention.

FIGURE 3. Visual acuity (LogMAR) before and after 5 min of periocular massage in the Chinese eye exercise (CE), facial massage roller (MR), automated eye massager (AM), and no-massage (control; CT) groups; data are mean and SD values.

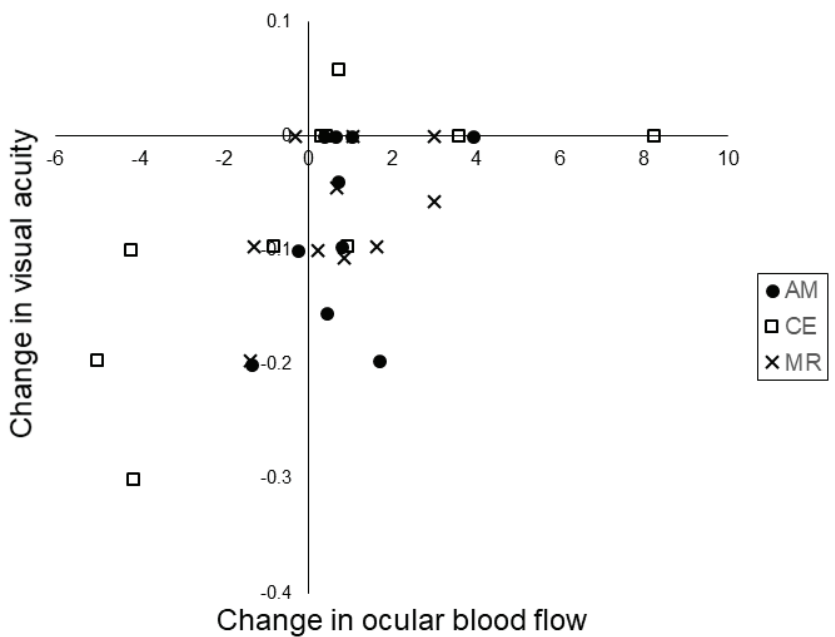

FIGURE 4. Relationship between changes in the ocular blood flow (measured as the mean blur rate) and visual acuity after 5 minutes of periocular massage in the CE, MR, and AM groups; no significant correlation was observed.

The OBF, visual acuity, and contrast sensitivity before and after the 60-day chronic massage period are summarized in Table 3. ANOVA did not reveal any significant effect. No significant correlation was observed in changes between the MBR and visual acuity or between the visual acuity and the change therein.

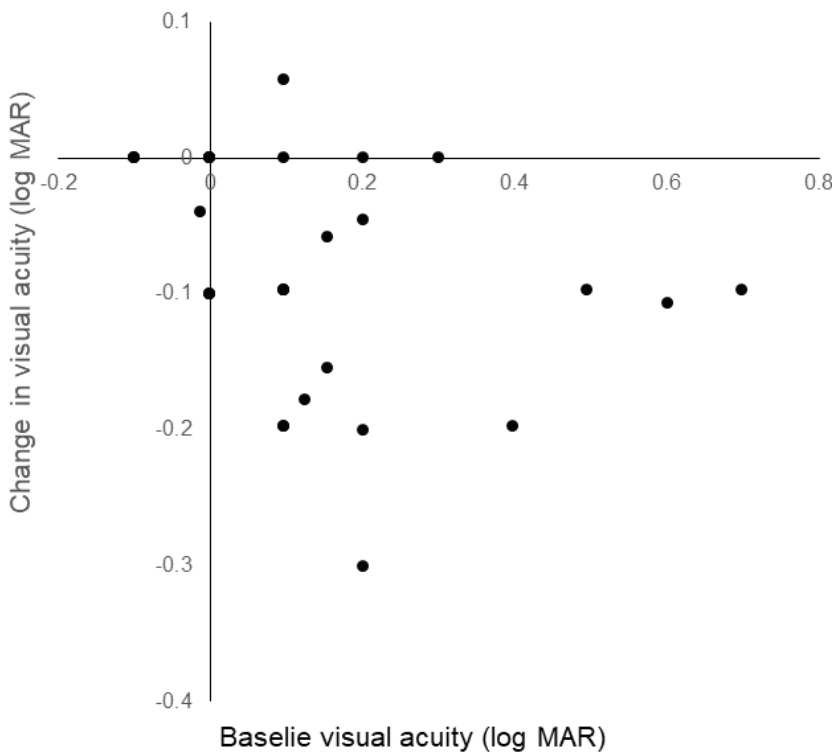

$\log M A R=$ logarithm of the minimum angle of resolution.

FIGURE 5. Relationship between the baseline visual acuity and the change in visual acuity after $5 \mathrm{~min}$ of periocular massage; there was a tendency for a significant correlation $(p<.7)$.

Supplemental paired $t$ tests of the pooled data obtained from the right eyes in the three massage groups $(n=30)$ showed a significant difference between before and after a 60-day massage period $(0.14 \pm 0.20$ vs. $0.075 \pm 0.22$ )

\section{DISCUSSION}

We examined the effects of short-term and long-term periocular massage on OBF and vision. A 5-min massage had a significant effect on the MBR of the OBF and the static visual acuity compared to the control condition. The findings suggested that periocular massage can improve OBF and visual function, with these effects varying with the type of massage. However, the factors responsible for improving vision remain unclear since no relationship was observed between changes in visual function and other variables. An acute effect was not observed for stiff ness variables of the ocular vessels. Effects of chronic periocular massage on OBF and visual function were not supported.

\section{Acute Effects}

The static visual acuity improved after 5 min of periocular massage from 0.14 to 
HAYASHI: PERIOCULAR MASSAGE FOR OCULAR BLOOD FLOW AND VISION

TABLE 3. Changes in Visual Functions and Ocular Circulation Before and After Two-Month Periocular Massage

\begin{tabular}{|c|c|c|c|c|c|c|}
\hline Group & & Visual Acuity & Cont. Sens. 10\% & $M B R$ & BOS & BOT \\
\hline \multirow[t]{2}{*}{$C E^{a}$} & Before & $0.23 \pm 0.25$ & $0.24 \pm 0.18$ & $25.0 \pm 3.8$ & $79.5 \pm 4.1$ & $57.5 \pm 4.5$ \\
\hline & After & $0.18 \pm 0.17$ & $0.28 \pm 0.16$ & $22.6 \pm 3.2$ & $79.3 \pm 4.3$ & $57.3 \pm 5.1$ \\
\hline \multirow[t]{2}{*}{$\mathrm{AM}^{\mathrm{a}}$} & Before & $0.06 \pm 0.09$ & $0.37 \pm 0.15$ & $27.0 \pm 5.5$ & $79.9 \pm 2.3$ & $58.1 \pm 4.3$ \\
\hline & After & $-0.06 \pm 0.05$ & $0.49 \pm 0.19$ & $22.7 \pm 4.4$ & $80.3 \pm 2.3$ & $55.8 \pm 2.7$ \\
\hline \multirow[t]{2}{*}{$\mathrm{CT}^{\mathrm{a}}$} & Before & $0.15 \pm 0.28$ & $0.29 \pm 0.21$ & $25.3 \pm 7.2$ & $81.1 \pm 2.8$ & $58.0 \pm 3.1$ \\
\hline & After & $0.17 \pm 0.24$ & $0.36 \pm 0.26$ & $25.0 \pm 5.6$ & $79.4 \pm 2.7$ & $57.9 \pm 2.7$ \\
\hline \multirow[t]{2}{*}{$M R^{b}$} & Before & $0.12 \pm 0.19$ & $0.35 \pm 0.21$ & $25.6 \pm 5.1$ & $80.9 \pm 2.8$ & $58.3 \pm 2.6$ \\
\hline & After & $0.11 \pm 0.30$ & $0.48 \pm 0.27$ & $3.9 \pm 5.8$ & $82.2 \pm 2.7$ & $59.2 \pm 3.2$ \\
\hline \multirow[t]{2}{*}{$\mathrm{MR}(\mathrm{Ctrl})^{\mathrm{b}}$} & Before & $0.18 \pm 0.30$ & $0.41 \pm 0.23$ & $27.6 \pm 5.3$ & $80.8 \pm 2.8$ & $58.0 \pm 2.5$ \\
\hline & After & $0.12 \pm 0.22$ & $0.46 \pm 0.24$ & $24.9 \pm 5.9$ & $81.6 \pm 4.3$ & $58.8 \pm 3.6$ \\
\hline
\end{tabular}

a In CE (Chinese eye exercise) and AM (automatic massager) groups, CT (control) was used as control intervention.

bIn MR (facial massage roller) group, control left eye (MR Ctrl) was used as control intervention.

Cont Sens = contrast sensitivity; $\mathrm{MBR}=$ mean blur rate (blood flow); BOS = blow-out score; BOT = blow-out time.

0.056 logMAR in three pooled massage groups, corresponding to decimal visual acuity value changing from 0.72 to 0.88 . This short-term effect indicates that the 5-min massage is an effective and easy method for improving vision. Eye fatigue associated with visual display terminals (including head-mounted displays) is currently a major issue that is being studied from various viewpoints, $(27,28)$ and the present findings indicate that a simple intervention allows the transient recovery of eye fatigue.

Applying periocular massage affects the OBF. In an anaesthetized rabbit model, optic nerve blood flow reportedly increased after 15 min of manual massage. (29) This may be consistent with the present findings, although that rabbit model differed markedly from the present human model. There was a measurable increase (about 4\%), but the change was much less than those observed during cycling and handgrip exercise (generally more than $10 \%) .(30,31)$ No relationship with changes in visual acuity was observed, and so the physiological and functional significance is unclear.

The mechanism via which periocular massage influences visual acuity is still unclear. The importance of acupuncture points was suggested by a randomized controlled trial that found an improvement in accommodative lag after one month of standard Chinese eye exercise, but not after applying eye exercise to sham locations. (32) However, no effect of acupuncture points was seen for the present short-term massage interventions, with the three types of periocular massage producing similar improvements in visual acuity. Periocular massage might acutely decrease the axial length of the eye, which relates to myopia. ${ }^{1,2)}$ Myopia occurs when the cornea and lens do not compensate for axial elongation. Massage pressure could potentially push the eyeball so as to decrease the axial length. However, the probability of this in the present study is expected to be low, since the periocular massage applied did not involving pushing the eyeball with pressure. Besides, if there was an effect of pressure by massage, the OBF would have been expected to decrease due to an acute increase in the external pressure on the vessels, whereas it actually increased after the massage. Therefore, the present findings are not considered to have been influenced by the axial length, although this should be confirmed in future research.

The effect of the visual acuity before massage on the change in visual acuity should also be considered. Although the correlation was not significant, a tendency was observed. The key to practical applications of periocular massage is that the acuity improves after massage in those who have poor visual acuity, since this study found that the visual acuity improved after massage in all of the four subjects who had more than 0.4 logMAR (i.e., worse than 0.4 decimal).

\section{Chronic Effects}

Previous studies have suggested that massage produces solely subjective 
changes, or only small or modest objective changes in the vision of children. $(8,9,10,11)$ A retrospective study found that Chinese eye exercise exerts a modest effect on relieving near-vision symptoms, but no remarkable effect on reducing myopia. One month of Chinese eye exercise reduced accommodative lag in children, whereas no effect was found in visual acuity. ${ }^{(32)}$ We recruited adult subjects in order to exclude the effects of growth that have been reported previously, ${ }^{(9)}$ but we still did not find significant effects of massage on vision and OBF.

We found no changes in OBF variables even when using pooled data. Ageing increases the stiffness of ocular vessels and decreases the MBR. ${ }^{23,24)}$ We anticipated that short-term periocular massage can improve OBF variables, but there is no evidence for the improvement of OBF by chronic periocular massage at present.

\section{Limitations}

There are three methodological limitations in the present study. First, it should be noted that participants in the present study were Asian people between the ages of 18 and 28 years, with relatively high rate of eyesight correction, limiting the extrapolations to other groups. No one is sure if the age range was old enough to obtain an effect from massage. Effect of age on myopia status has been reported. ${ }^{(1,4)}$ Also the type of myopia (onset of youth, early adult, or late adult) cannot be ruled out; we do not consider this variable in the present study. A wider variety of participants are needed for future studies.

Second, we are not able to control the speed, area, and intensity of periocular massage, as well as massage to other areas, although the present methodology may be a reasonable method at this time, when we consider the actual method of massage.

Third, we used a 60-day period for the long-term experiment. This period can probably not be long enough to show any effects of massage. The period of 60 days was selected since a previous study, using one month of the Chinese eye exercise, reported improvement in accommodative lag exercise, (32) and we reported changes in skin blood flow response to temperature stimuli after five-week facial skin massage. (19) Taken together, it was thought that a 60-day observation period was appropriate.

Clinical significance of the change in visual acuity should also be noted, though the transient improvement after the shortterm massage was statistically significant. As in a previous study, ${ }^{(26)}$ the logarithmic transformation was performed and the analysis was statistically appropriate. On the other hand, since visual acuity is measured as a step-wise variable, we have to be careful when judging the change as a clinically significant change.

\section{CONCLUSION}

We have investigated the acute and chronic effects of applying three types of periocular massage on visual acuity, contrast sensitivity, and OBF. Performing 5-min periocular massage showed significant interactions in time and massage effects on visual acuity in CE and AM groups, and on OBF in AM group, while two-month massage period exerted no significant effects. No significant relationship was found between OBF and visual acuity changes. These results suggest that short-term periocular massage with Chinese eye exercise and automated eye massager can improve OBF and visual acuity, although no causal relationship was supported.

\section{ACKNOWLEDGMENTS}

We thank MTG Co. Ltd. (Nagoya, Japan) for providing the facial massage roller and the automated eye massager. MTG Co. Ltd. did not influence this study in any way.

\section{CONFLICT OF INTEREST NOTIFICATION}

The authors declare there are no conflicts of interest.

\section{COPYRIGHT}

Published under the CreativeCommons Attribution-NonCommercial-NoDerivs 3.0 License.

\section{REFERENCES}

1. Meng W, Butterworth J, Malecaze F, Calvas P. Axial length of myopia: a review of current research. Ophthalmologica. 2011;225(3):127-134.

2. Morgan IG, Ohno-Matsui K, Saw S-M. Myopia. Lancet. 2012;379(9827):1739-1748. 
3. Pan CW, Ramamurthy D, Saw S-M. Worldwide prevalence and risk factors for myopia. Ophthalmic Physiol Opt. 2012;32(1):3-16.

4. Liang YB, Friedman DS, Wong TY, Zhan SY, Sun LP, Wang JJ, et al. Prevalence and causes of low vision and blindness in a rural Chinese adult population: the Handan Eye Study. Ophthalmology. 2008;115(11):1965-1972.

5. Buch $\mathrm{H}$, Vinding $\mathrm{T}$, la Cour $\mathrm{M}$, Appleyard $\mathrm{M}$, Jensen GB, Nielsen NV. Prevalence and causes of visual impairment and blindness among 9980 Scandinavian adults. Ophthalmology. 2004;111(1):53-61.

6. Cooper J, Schulman E, Jamal N. Current status on the development and treatment of myopia. Optometry. 2012;83(5):179-199.

7. Gwiazda J. Treatment options for myopia. Optom Vis Sci. 2009;86(6):624-628.

8. Kang M-T, Li S-M, Peng X, Li L, Ran A, Meng B, et al. Chinese eye exercises and myopia development in school age children: a nested case-control study. Sci Rep. 2016;6(1):1-8.

9. Cha H-Y, Jung A-R, Kang B-k, Song J-H, Jung JH, Cheon JH, et al. The effect of periocular acupressure with a medical massager for myopia children. J Korean Med. 2016;37(2):53-61.

10. Lin Z, Vasudevan B, Jhanji V, Gao TY, Wang NL, Wang $Q$, et al. Eye exercises of acupoints: their impact on refractive error and visual symptoms in Chinese urban children. BMC Complement Altern Med. 2013;13(1):1-9.

11. Östberg $\bigcirc, Y$ Horie, Y Feng. On the merits of ancient Chinese eye acupressure practices. Appl Ergon. 1992;23(5):343-348.

12. Lippi G, Henry BM, Bovo C, Sanchis-Gomar F. Health risks and potential remedies during prolonged lockdowns for coronavirus disease 2019 (COVID-19). Diagnosis. 2020;7(2):85-90.

13. Ryskulova A, Turczyn K, Makuc DM, Cotch MF, Klein RJ, Janiszewski R. Self-reported age-related eye diseases and visual impairment in the United States: results of the 2002 National Health Interview Survey. Am J Public Health. 2008;98(3): 454-461.

14. Wen SW, Wong CHY. Aging- and vascular-related pathologies. Microcirculation. 2019;26(2):e12463.

15. World Health Organization. Universal eye health: a global action plan 2014-2019. Geneva, Switzerland: WHO Press; 2013.

16. Delaey C, Van De Voorde J. Regulatory mechanisms in the retinal and choroidal circulation. Ophthalmic Res. 2000;32(6):249-256.

17. Hayashi N, Ikemura T, Someya N. Changes in ocular flow induced by hypo- and hypercapnia relate to static visual acuity in humans. Eye Rep. 2011;1(1):e8.

18. Franklin NC, Ali MM, Robinson AT, Norkeviciute E, Phillips SA. Massage therapy restores peripheral vascular function after exertion. Arch Phys Med Rehabil. 2014;95(6):1127-1134.
19. Miyaji A, Sugimori K, Hayashi N. Short- and longterm effects of using a facial massage roller on facial skin blood flow and vascular reactivity. Complement Ther Med. 2018;41:271-276.

20. Weerapong P, Hume PA, Kolt GS. The mechanisms of massage and effects on performance, muscle recovery and injury prevention. Sports Med. 2005;35(3):235-256.

21. Takahashi H, Sugiyama T, Tokushige H, Maeno T, Nakazawa T, Ikeda T, et al. Comparison of CCDequipped laser speckle flowgraphy with hydrogen gas clearance method in the measurement of optic nerve head microcirculation in rabbits. Exp Eye Res. 2013;108:10-15.

22. Wang L, Cull GA, Piper C, Burgoyne CF, Fortune B. Anterior and posterior optic nerve head blood flow in nonhuman primate experimental glaucoma model measured by laser speckle imaging technique and microsphere method. Invest Ophthalmol Vis Sci. 2012;53(13):8303-8309.

23. Kobayashi T, Shiba, T, Kinoshita A, Matsumoto T, Hori Y. The influences of gender and aging on optic nerve head microcirculation in healthy adults. Sci Rep. 2019;9(1):1-8.

24. Miyaji A, Ikemura T, Hayashi N. Effect of aging on the blowout time in various ocular vessels. Aging Sci. 2016;4:148.

25. Sugiyama T. Basic technology and clinical applications of the updated model of laser speckle flowgraphy to ocular diseases. Photonics. 2014;1(3):220-234.

26. Holladay JT. Visual acuity measurements. J Cataract Refract Surg. 2004;30(2):287-290.

27. Glimne S, Brautaset R, Österman C. Visual fatigue during control room work in process industries. Work. 2020;65(4):903-914.

28. Guo J, Weng D, Zhang Z, Liu Y, Duh HB-L, Wang Y. Subjective and objective evaluation of visual fatigue caused by continuous and discontinuous use of HMDs. J Soc Inf Disp. 2019;27(2):108-119.

29. Jay WM, Aziz MZ, Green K. Effect of digital massage on intraocular pressure and ocular and optic nerve blood flow. Acta Ophthalmol. 1986;64(1):58-62.

30. Hayashi N, Ikemura T, Someya N. Effects of dynamic exercise and its intensity on ocular blood flow in humans. Eur J Appl Physiol. 2011;111(10):2601-2606.

31. Ikemura T, Someya N, Hayashi N. Autoregulation in the ocular and cerebral arteries during the cold pressor test and handgrip exercise. Eur J Appl Physiol. 2012;112(2):641-646.

32. Li SM, Kang MT, Peng XX, Li SY, Wang Y, Li L, et al. Efficacy of Chinese eye exercises on reducing accommodative lag in school-aged children: a randomized controlled trial. PLoS One. 2015;10(3):e0117552.

Corresponding author: Naoyuki Hayashi, PhD, Faculty of Sport Sciences, Waseda University, Mikajima 2-579-15, Tokorozawa 359-1192, Saitama, Japan

E-mail: naohayashi@waseda.jp 